\title{
Medical Image of the Week: Right Middle Lobe Syndrome
}

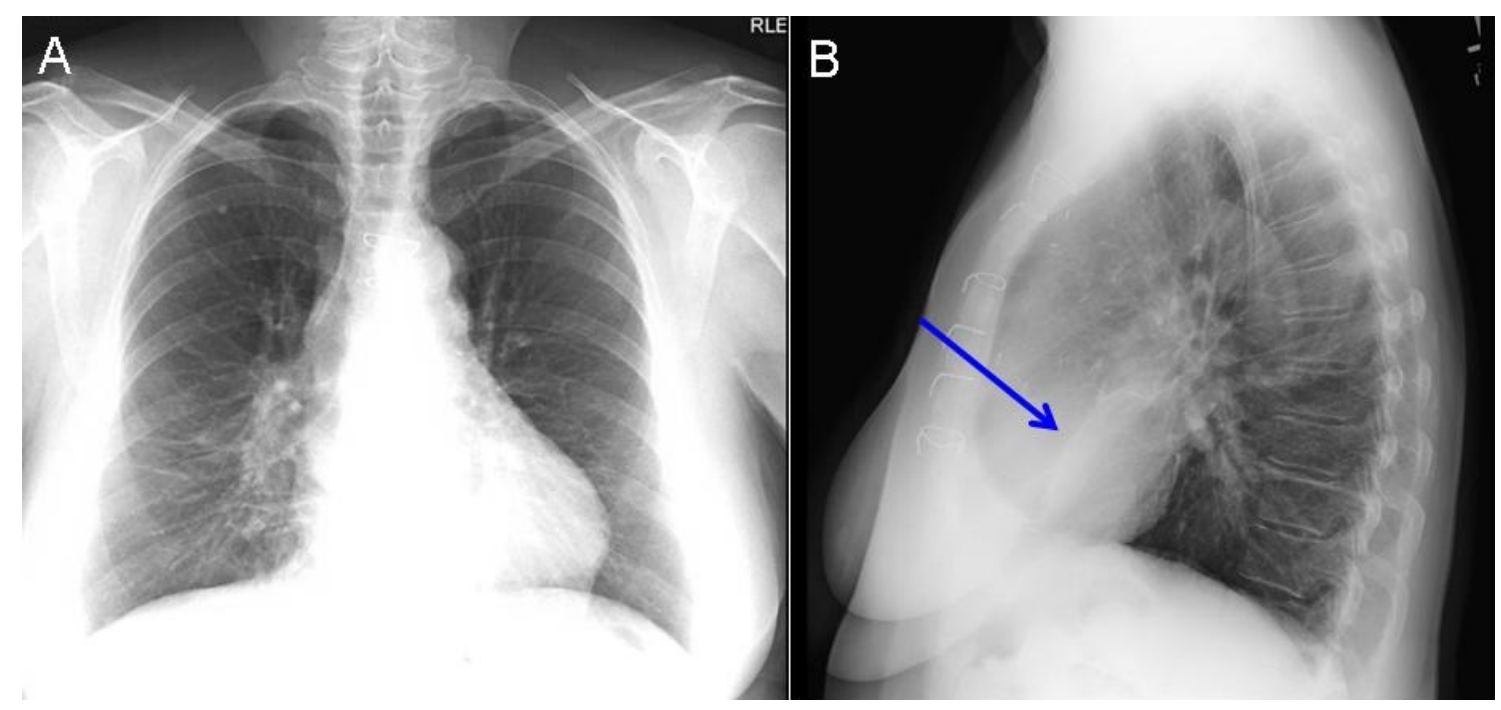

Figure 1. Panel A: PA chest x-ray showing calcified mediastinal lymphadenopathy. Panel B: Lateral view showing a triangle of increased density between the minor fissure and the lower half of the major fissure seen on the lateral view (blue arrow) .

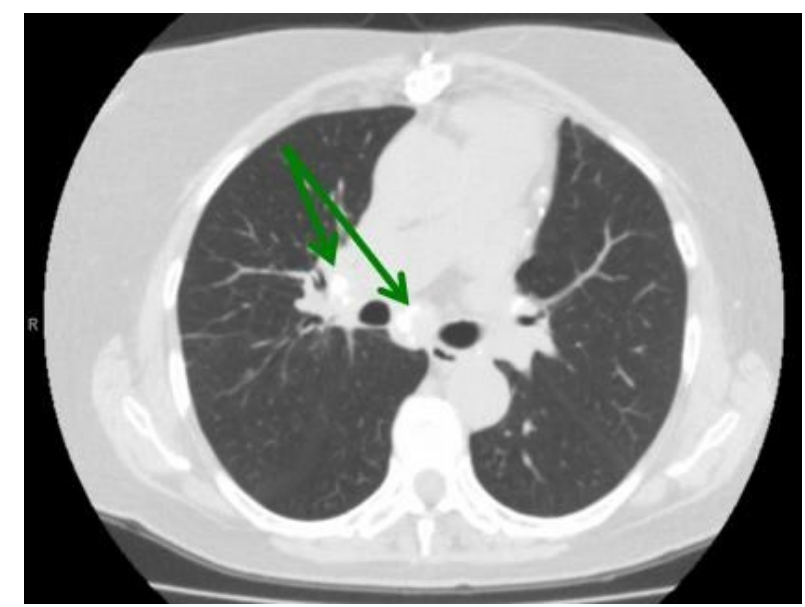

Figure 2. Image from the thoracic CT scan in lung windows showing calcified mediastinal lymph notes (green arrows).

A 73 year-old woman, a lifetime non-smoker, presented to the pulmonary clinic with chronic dyspnea on exertion and cough. Physical exam was unremarkable and pulmonary function testing showed normal spirometry. A chest radiograph revealed calcified mediastinal adenopathy and increased density in the right middle lobe region (Figure 1). A computed tomography scan of the chest revealed significant narrowing of the right middle lobe bronchus with partial atelectasis and prominent calcified 
mediastinal lymphadenopathy (Figure 2). Bronchoscopy showed no endobronchial lesions but there was evidence of extrinsic compression surrounding the right middle lobe orifice. An endobronchial biopsy revealed noncaseating granulomas.

Bronchoscopy cultures and cytology were negative and this was presumed to be from a previous infection with histoplasmosis given the patient's long-term residence in an endemic area. Given chronic narrowing of right middle lobe bronchus with persistent atelectasis of the right middle lobe, the patient was diagnosed with right middle lobe syndrome. She was started on combination therapy with a long-acting beta agonist and inhaled corticosteroid with complete resolution of her symptoms.

Right middle lobe syndrome (RMLS) is defined as recurrent or chronic atelectasis of the right middle lobe. Although more commonly described in children, it is becoming more prevalent in adults with a predilection for women. There are two distinct types of pathophysiology- obstructive and non-obstructive. Obstructive pathophysiology is defined when there is an endobronchial lesion or extrinsic compression of the middle lobe bronchus by lymphadenopathy (as in our case) or a tumor. Non-obstructive pathophysiology occurs when there is recurrent infection or inflammation leading to bronchiectasis and scarring. Certain anatomical characteristics, including the acute take-off angle of the right middle lobe bronchus create poor conditions for drainage and collateral ventilation (1).

Symptoms of RMLS include chronic or recurrent cough, dyspnea, wheezing and recurrent infections. High resolution computed tomography of the chest is the gold standard for imaging, as this will show narrowing of the right middle lobe orifice along with etiologies of extrinsic compression (Figure 2). Patients suspected of having RMLS warrant a bronchoscopy to evaluate for patency of right middle lobe bronchus, to exclude malignancy and for evaluation of infectious etiologies (1). The treatment of RMLS includes bronchodilator therapy along with mucolytics, chest physiotherapy and antibiotics if bronchiectasis is problematic. Lobectomy may be warranted if malignancy is diagnosed, aggressive medical management fails or hemoptysis occurs (2).

Elaine A. Cristan, MD and Linda Snyder, MD

Department of Medicine

Division of Pulmonary, Critical Care, Sleep and Allergy Medicine

Banner University Medical Center

Tucson, AZ USA

\section{References}

1. Gudbjartsson T, Gudmundsson G. Middle lobe syndrome: a review of clinicopathological features, diagnosis and treatment. Respiration. 2012;84(1):80-6. [CrossRef] [PubMed]

2. Einarsson JT, Einarsson JG, Isaksson H, Gudbjartsson T, Gudmundsson G. Middle lobe syndrome: a nationwide study on clinicopathological features and surgical treatment. Clin Respir J. 2009 Apr;3(2):77-81. [CrossRef] [PubMed] 